\section{Reduced physical activity in lymphangioleiomyomatosis compared with COPD and healthy controls: disease- specific impact and clinical correlates}

\section{Abstract \\ Rationale Sporadic lymphangioleiomyomatosis (LAM) is an orphan lung disease for which daily physical activity has not been studied so far and it is unclear whether a disease-specific impact beyond airflow limitation exists. Clinical correlates indicating reduced physical activity in addition to established parameters like airflow limitation and hypoxaemia are largely undetermined.}

Method We measured physical activity (steps per day, SPD; physical activity level, PAL; minutes of moderate activity, MMA) in 34 women with LAM, $32 \mathrm{FEV}_{1}$-matched female patients with COPD and 15 age-matched healthy women for 1 week using an accelerometer. In addition, we assessed lung function measurements, questionnaires for generic and respiratory health status (12-Item Short Form Survey, SF-12; St. George's Respiratory Questionnaire, SGRQ), dyspnoea (modified Medical Research Council dyspnoea scale, $\mathrm{mMRC}$ ) and fatigue (Multidimensional Fatigue Inventory, MFI-20). Results Patients with LAM (mean age 52.7 years, mean $F E V, 62.7 \%$ predicted) showed reduced SPD, PAL and MMA ( $p<0.01)$ compared with healthy controls and reduced MMA ( $p=0.032$ ) compared with female patients with COPD (mean age 65.2 years, mean FEV $162.6 \%$ predicted). In multivariate regression analyses, adjusting for $\mathrm{FEV}_{1}$ and long-term oxygen therapy, either generic health status (SF-12 physical health) or fatigue (MFI-20) were the strongest independent predictors for SPD in patients with LAM ( $p=0.006$ and $p=0.004$, respectively). Conclusions Physical activity in daily life is substatially reduced in LAM, when compared with healthy controls and COPD - indicating a disease specific impact. The regular assessment of fatigue and generic health status may improve disease management in LAM by taking daily physical activity of patients with LAM more adequately into account.

Sporadic lymphangioleiomyomatosis (LAM) is a rare lung disease that affects almost exclusively women. It is characterised by cyst formation leading to airflow limitation, ventilatory limitations and gas exchange impairment, resulting in dyspnoea and exercise intolerance. ${ }^{1}$ Daily physical activity is an important clinical outcome parameter in common lung diseases, such as COPD. ${ }^{2}$ However, rare lung diseases such as LAM have not been studied so far and a disease-specific contribution to daily physical activity beyond airflow limitation is uncertain. In LAM, fatigue is a highly prevalent feature that has been overlooked in the past ${ }^{3}$ and that might be associated with levels of daily physical activity in this debilitating disease, just like health status and dyspnoea. Here, we hypothesised that physical activity in patients with LAM is substantially lower than in healthy controls but comparable with patients with COPD. In addition, we hypothesised that clinical variables other than airflow limitation and hypoxaemia might be associated with physical activity in patients with LAM.

We measured physical activity in 34 female patients with LAM, 32 female patients with COPD and 15 healthy women for 1 week using an activity monitor (SenseWear Armband; BodyMedia, Pittsburgh, USA) measuring steps per day (SPD), physical activity level (PAL) and minutes of at least moderate activity per day (MMA), as previously described. $^{2}$ We recruited patients with LAM (mean age 52.7 years, mean $\mathrm{FEV}_{1}$ $62.6 \%$ predicted, long-term oxygen therapy (LTOT): $n=12(35 \%))$ during the annual meeting of the German LAM patient group. Patients with COPD (mean age 65.2 years, mean $\mathrm{FEV}_{1} 62.7 \%$ predicted, LTOT: $n=6(19 \%))$ were derived from an existing cohort ${ }^{4}$ and matched for $\mathrm{FEV}_{1}$ and body mass index (BMI). Dyspnoea and health status were similar between groups. Healthy controls were recruited from our institutional database ${ }^{5}$ and matched for age and BMI (see online supplementary table E1 for detailed characteristics). All participants provided written informed consent (ethics approval AZ 038/12 II/AZ 185/08; German Clinical Trials Register Number DRKS00006170). Lung function parameters of patients with LAM were taken from the last available lung function report. Participants filled out questionnaires for generic and respiratory health status (12-Item Short Form Survey, SF-12; St. George's Respiratory Questionnaire, SGRQ), dyspnoea (modified Medical Research Council dyspnoea scale, mMRC) and fatigue (Multidimensional Fatigue Inventory, MFI- $20^{6}$ ) at home and sent them back together with the activity monitors at the end of the measurement.

Compared with healthy controls, each of the three physical activity parameters (SPD, PAL and MMA) was significantly reduced in patients with LAM (figure 1). Unadjusted comparison between patients with LAM and COPD showed reduced MMA in LAM (figure 1), despite the significant age difference and equal dyspnoea and health status scores. Adjusted for age and LTOT as possible confounders, multivariate regression analyses showed significantly lower MMA and PAL in patients with LAM compared with COPD $(\mathrm{p}=0.006$ and $\mathrm{p}=0.005$, respectively),
A

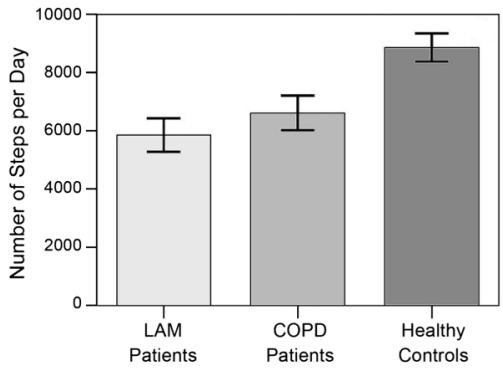

B

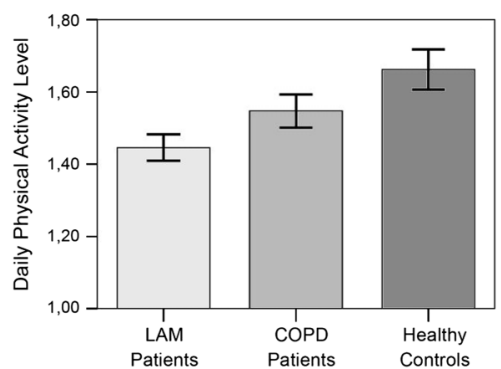

C

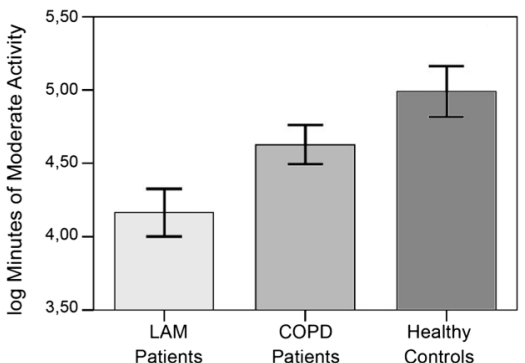

Figure 1 Physical activity of patients with lymphangioleiomyomatosis (LAM) (bright bar) compared with patients with COPD (light grey) and healthy controls (dark grey) expressed as mean \pm SE. (A) Number of steps per day $(5852 \pm 3327$ vs $6616 \pm 3351$ vs $8863 \pm 1884$; reduction by $12 \%$ vs $34 \% ; p=0.360$ and $p=0.002$, respectively). (B) Daily physical activity level $(1.45 \pm 0.21$ vs $1.54 \pm 0.26$ vs $1.66 \pm 0.22 ;$ reduction by $24 \%$ vs $32 \%$; $p=0.087$ and $p=0.002$, respectively). (C) Log transformed minutes of at least moderate activity per day (corresponding medians (IQR) were 61 (26147) vs 107 (46-78) vs 153 (97-207); reduction of the median by $43 \%$ vs $60 \% ; p=0.032$ and $p=0.006$, respectively). 
whereas SPD were not significantly lower $(p=0.152) \quad$ (online supplementary table E2). A potential explanation for this observation might be that patients with LAM walk at lower intensities compared with patients with COPD.

Airflow limitation, health status, dyspnoea and fatigue scores correlated best with SPD as outcome variable (online supplementary table E3). In multivariate regression analyses, adjusting for LTOT and $\mathrm{FEV}_{1}$, either SF-12 physical health or MFI-20 were the strongest independent predictors for SPD in patients with LAM (total $\mathrm{R}^{2}=0.476$, partial $\mathrm{R}^{2}=0.178$, $\mathrm{p}=0.006$ and total $\mathrm{R}^{2}=0.449$, partial $\mathrm{R}^{2}=0.207, \mathrm{p}=0.004$, respectively) (online supplementary table E4).

Measures on exercise capacity (eg, 6 min walking test) and associated hypoxaemia might explain further variance in the data. The lack of these measurements is a clear limitation of our study.

We show for the first time that physical activity in daily life is substantially reduced in LAM patients, when compared with healthy controls and COPD patients with similar functional limitation, dyspnoea and health status-indicating a disease-specific impact. The underlying processes, however, are to be elucidated in further studies. Although airflow limitation and hypoxaemia - as indicated by $\mathrm{FEV}_{1}$ and LTOT-are well-established predictors for impaired physical activity, we propose to include fatigue and generic health status into the standard clinical assessment of patients with LAM. Awareness and measurement of both features will help to improve adequate disease management in this debilitating disease.

Thomas Bahmer, ${ }^{1}$ Henrik Watz, ${ }^{2}$ Benjamin Waschki, ${ }^{1}$ Marco Gramm ${ }^{2}$ Helgo Magnussen, ${ }^{2}$ Klaus F Rabe, Hubert Wirtz, ${ }^{3}$ Detlef Kirsten, ${ }^{1}$ Anne Kirsten ${ }^{2}$

${ }^{1}$ LungenClinic Grosshansdorf, Airway Research Center North, Member of the German Center for Lung Research, Grosshansdorf, Germany

${ }^{2}$ Pulmonary Research Institute at LungenClinic Grosshansdorf, Airway Research Center North, Member of the German Center for Lung Research, Grosshansdorf, Germany

${ }^{3}$ Pneumology Department, Universitätsklinikum Leipzig AöR, Leipzig, Germany

Correspondence to Thomas Bahmer, LungenClinic Grosshansdorf, Woehrendamm 80, 22927

Grosshansdorf, Germany; t.bahmer@lungenclinic.de

Acknowledgements The authors thank the patients of German LAM foundation ('LAM Selbsthilfe e.V.') for supporting this study. We thank Daniela Beissel for her excellent assistance with study logistics.

Contributors TB had full access to all of the data and takes full responsibility for the integrity of the data and the accuracy of the data analysis. TB: contributed to statistical analysis and interpretation of the data and drafting of the manuscript. HW: contributed to conception and design of the study, acquisition, analysis and interpretation of data and drafting of the manuscript. BW: contributed to statistical analysis and interpretation of the data and drafting of the manuscript. MG: contributed to statistical analysis and critically revised the manuscript. HM: contributed to conception and design of the study and critically revised the manuscript. KFR: contributed to conception and design of the study and critically revised the manuscript. HW: contributed to conception and design of the study and critically revised the manuscript. DK: contributed to conception and design of the study and critically revised the manuscript. AK: contributed to conception and design of the study, analysis and interpretation of data and drafting of the manuscript. All coauthors critically revised the article and gave final approval of this version to be published.
Funding The work was supported by

'Wissenschaftliche Arbeitsgemeinschaft zur Therapie von Lungenerkrankungen' (WATL e.V.).

Competing interests None declared.

Ethics approval Ethics Committee Schleswig-Holstein, Bad Segeberg, Germany; AZ 038/12 (II) and AZ 185/08.

Provenance and peer review Not commissioned; externally peer reviewed.

- Additional material is published online only. To view please visit the journal online (http://dx.doi.org/10.

1136/thoraxjnl-2015-207852)

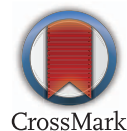

To cite Bahmer T, Watz H, Waschki B, et al. Thorax 2016;71:662-663.

Received 17 September 2015

Revised 14 January 2016

Accepted 9 February 2016

Published Online First 11 March 2016

Thorax 2016;71:662-663.

doi:10.1136/thoraxjnl-2015-207852

\section{REFERENCES}

1 Baldi BG, Albuquerque AL, Pimenta SP, et al. Exercise performance and dynamic hyperinflation in lymphangioleiomyomatosis. Am J Respir Crit Care Med 2012:186:341-8.

2 Watz $\mathrm{H}$, Waschki B, Meyer $\mathrm{T}$, et al. Physical activity in patients with COPD. Eur Respir J 2009;33:262-72.

3 Cohen MM, Pollock-BarZiv S, Johnson SR. Emerging clinical picture of lymphangioleiomyomatosis. Thorax 2005;60:875-9.

4 Waschki B, Kirsten AM, Holz O, et al. Disease progression and changes in physical activity in patients with COPD. Am J Respir Crit Care Med 2015;192:295-306.

5 Cockayne DA, Cheng DT, Waschki B, et al. Systemic biomarkers of neutrophilic inflammation, tissue injury and repair in COPD patients with differing levels of disease severity. PLOS ONE 2012;7:e38629.

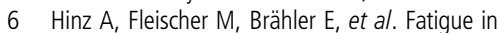
patients with sarcoidosis, compared with the general population. Gen Hosp Psychiatry 2011;33:462-8. 